\title{
fMRI-Compatible Robotic Interfaces with Fluidic Actuation
}

\author{
Ningbo Yu ${ }^{1}$, Christoph Hollnagel ${ }^{1}$, Armin Blickenstorfer ${ }^{2}$, Spyros Kollias ${ }^{2}$, Robert Riener ${ }^{1}$ \\ ${ }^{1}$ Sensory-Motor Systems Lab, ETH and University Zurich, Switzerland \\ ${ }^{2}$ Institute of Neuroradiology, University Hospital Zurich, Switzerland
}

\begin{abstract}
Actuation is a major challenge in the development of robotic systems intended to work in functional Magnetic Resonance Imaging (fMRI) procedures, due to the high magnetic fields and limited space in the scanner. Fluidic actuators can be made fMRI-compatible and are, thus, promising solutions. In this work we developed two robotic interface devices, one with hydraulic and another with pneumatic actuation, to control one degree-of-freedom translational movements of a user that performs fMRI tasks. Due to the fMRI-compatibility restrictions, special materials were used for the endeffector which works in the scanner bore, and active components such as the control valves and pressure sensors, had to be placed far away from the endeffector with long transmission lines in between. Therefore, the two fMRI-compatible setups differed from conventional fluidic actuation systems and brought control difficulties. Both systems have been proved to be fMRI-compatible and yield no image artifacts in a 3T scanner. Passive as well as active subject movements were realized by classical position and impedance controllers. With the hydraulic system we achieved smoother movements, higher position control accuracy and improved robustness against force disturbances than with the pneumatic system. In contrast, the pneumatic system was back-drivable, showed faster dynamics with relatively low pressure, and allowed force control. Furthermore, it is easier to maintain and does not cause hygienic problems after leakages. In general, pneumatic actuation is favorable for fast or force-controlled applications, whereas hydraulic actuation is recommended for applications that require high position accuracy, or slow and smooth movements.
\end{abstract}

\section{INTRODUCTION}

Robotic systems and devices that are compatible with magnetic resonance imaging (MRI) technique find wide range of applications in academic and industrial fields [1, 2]. Functional MRI (fMRI) is an advanced research and clinical tool in neuroscience. An fMRI-compatible robot could perform well controlled and reproducible sensorimotor tasks, while the subject's motor interactions with the robot are recorded by fMRI procedures and translated into brain images (Fig. 1). Therefore, fMRI-compatible robots can be applied with fMRI procedures to map brain functions [3, 4], investigate human motor control $[5,6]$, monitor rehabilitation induced cortical reorganization in neurological patients [7], etc. Such kind of fMRI-robotic systems could provide insights into the cortical reorganization mechanism after damage to the nervous system, offer a better understanding of therapy-induced recovery, and help to derive more efficient rehabilitation strategies.

To construct fMRI-compatible devices is rather challenging. First, the device must not disturb the scanner magnetic fields and ensure image quality. Second, the device should function properly in the scanner room. Third, the device is compact to fit into the scanner bore with the diameter of $55 \sim 70 \mathrm{~cm}[1,3]$.

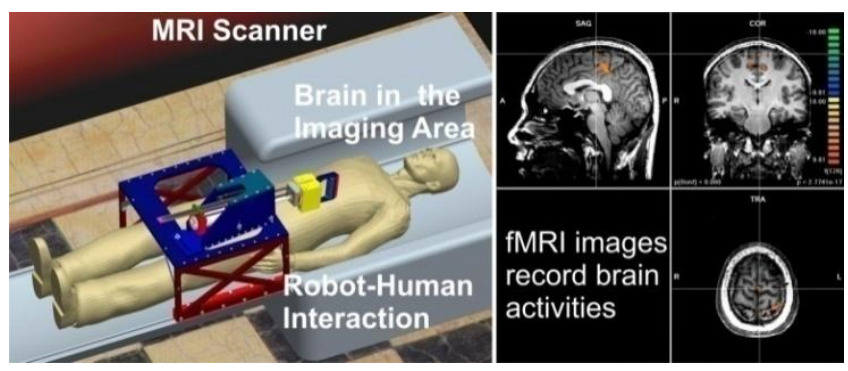

Fig. 1 fMRI-compatible robot working with fMRI procedures

The strong magnetic fields limit the choice of materials, sensors and actuators to be used in the MRI environment. Stiff polymer materials are a good alternative of magnetic metals for applications in the scanner environment. Sensors and actuators using strong electrical currents should also be avoided. Electrical components may be brought into the scanner environment if their electrical signals are of low frequency and low amplitude, and if the components are placed at a certain distance from the scanner and/or they are shielded [5, 6, 8]. Sensors with optical principles have been employed to measure position $[6,9]$, force / torque $[6,10,11]$.

Typical fMRI-compatible actuation technologies are based on hydraulic or pneumatic principles, special electromagnetic principles, shape memory alloys, contractile polymers, piezoelectric actuation, materials with magnetostriction properties, electro-rheological fluids (ERFs), or bowden cables [1, 2, 12, 13]. Among these working principles, fluidic actuations are promising solutions for fMRI-compatible robots that are intended to perform defined functional movement tasks, because 1) the fluids are magnetically inert in nature and the moving endeffector can be made fMRI-compatible, 2) the power can be generated distantly from the endeffector and sent to the endeffector inside the scanner via transmission hoses, 3) the actuators can provide large movement ranges and large forces, 4) the force-to-mass ratio is high, and 5) the transmission can be made flexible so that they can be placed adaptively to the work environment $[2,12]$.

In literature, many efforts have been made for the application of pneumatic actuation technologies to fMRI-compatible robotic systems [14] and devices [4, 15, 16]. Hydrostatic 
actuation was applied in master-slave setups to interact with human[9] or to position a forceps for surgery [17]. Reported problems were leakages, resulting in pollution, performance degeneration, and entrance of air bubbles. Furthermore, image deterioration occurred due to the high magnetic susceptibility of materials used for the systems [17, 18].

Traditional hydraulic or pneumatic actuation techniques cannot be directly transferred to fMRI-compatible applications. The fluid power generators, i.e., hydraulic or pneumatic compressors, consist of ferromagnetic materials. They must be placed outside of the scanner room for safety reason. Control valves are normally actuated by magnetically driven solenoids. Furthermore, valves and pressure sensors also contain ferromagnetic materials. Thus, they must be positioned far away from the scanner and the endeffector to avoid electromagnetic interferences causing malfunction and/or image artifacts. Therefore, long hoses have to be used to transmit the fluid power from the compressor to the control valves and then to the endeffector.

This arrangement results in several challenges for both construction and control. First, the endeffector must be made of fMRI-compatible materials so that it can work close to or inside the scanner bore. This can result in friction and stiffness problems at the fluidic cylinder, which is required to transfer fluidic pressure into force and motion. Second, valves and pressure sensors are distant from the endeffector, causing delay and measurement inaccuracies. Third, long hoses result in high inertia and compliance. Fourth, the system will interact with the user, so that the working pressure must be limited to ensure safety. Reduced pressure may also increase the compliance of the system. Finally, position and force sensors used inside the MRI scanner must be made MRI-compatible, which may reduce their signal quality. The mechatronic setup, including sensor, actuator and controller must be able to cope with these challenges and work in an, accurate, stable and robust way.

In this work two comparable robotic interface devices with hydraulic and pneumatic actuation respectively, were developed and implemented to control a translational one degree of freedom movement for fMRI studies. The interface devices are equipped with fMRI-compatible position and force sensors. Position and impedance/admittance controllers were realized to achieve active as well as passive subject movements, which are both required to investigate different fMRI-relevant motion tasks. The two systems were evaluated and compared with respect to control performance. Furthermore, both manipulandum systems were examined for MRI-compatibility in a 3 Tesla MRI scanner.

\section{DeSign AND REALIZATION OF THE FMRI-COMPATIBLE ROBOTIC SYSTEMS}

\section{A. Design Considerations and System Structure}

According to the prospective clinical applications, the design considerations are defined as:

- fMRI-compatible;

- Haptic interaction force and position measurement;

- Movement range: $0 \ldots 20 \mathrm{~cm}$;
- Actuator velocity range: $-15 \ldots 15 \mathrm{~cm} / \mathrm{s}$;

- Actuator force range: $-100 \ldots 100 \mathrm{~N}$;

- Subject passive movement: guide the subject's hand to follow a designed position curve;

- Subject active movement: simulate a virtual spring so that the subject can push or pull against the system.

The fluidic system structure was taken as in Fig. 2.

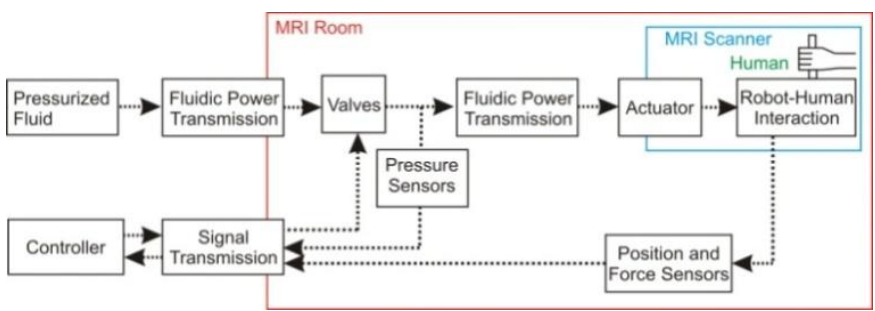

Fig. 2 Concept of fluidic-actuated robots to work with fMRI

\section{B. Material Consideration and Measurement Principles}

The materials put inside or close to the MRI scanner must have low magnetic susceptibility and low electric conductivity. Therefore, PET and PVC plastic were taken as the main construction material for frames and mechanical adapters. Nevertheless, metals have to be used for some parts required to be stiff, such as cylinders that will work under high pressure and force. Both cylinders were specially manufactured, with aluminum being the housing material. The piston of the pneumatic cylinder is made of PET, while that of the hydraulic cylinder is made of bronze to sustain the higher forces due to the significantly higher pressures. Both aluminum and bronze have low magnetic susceptibilities.

Table 1 Physical properties of several materials

\begin{tabular}{|l|c|c|}
\hline Material & $\begin{array}{c}\text { Magnetic } \\
\text { susceptibility }\end{array}$ & $\begin{array}{l}\text { Electrical conductivity } \\
{\left[\mathrm{m} / \Omega \mathrm{mm}^{2}, \text { or } 10^{6} \mathrm{~s} / \mathrm{m}\right]}\end{array}$ \\
\hline Bronze $\left(\mathrm{CuSn} 8^{*}\right)$ & $-0.879 \times 10^{-6}$ & 7.5 \\
\hline Zinc & $-15.7 \times 10^{-6}$ & 16.6 \\
\hline Brass & $-8.63 \times 10^{-6}$ & 17 \\
\hline Aluminum & $20.7 \times 10^{-6}$ & 36 \\
\hline Copper & $-9.63 \times 10^{-6}$ & 57 \\
\hline Drinking water & $-9.05 \times 10^{-6}$ & $0.05 \ldots 5 \times 10^{-2}$ \\
\hline Nickel & 600 & 14.4 \\
\hline Iron & 200000 & 9.9 \\
\hline
\end{tabular}

*: values provided by the supply company.

Both manipulandum systems are equipped with one force and two position sensors. The force sensor consists of three optical fibers, one with emitting laser light and two with receiving laser light [19]. When a pull or push force is applied to the handbar, the emitting fiber is slightly displaced, thus, changing the light intensities in the two receiving fibers. The measured force is a function of the ratio of light intensities $I_{l}$ and $I_{2}$. Laser signals $I_{1}$ and $I_{2}$ are sent out via glass fibers, converted to voltage signal by the processing circuit, and then read into the control computer. An optical encoder measures the handbar position, and a potentiometer works as a redundant position sensor for safety consideration.

\section{Fluidic Actuators}

The oil used in hydraulic actuation is Orcon Hyd 32, which is 
accepted as a lubricant with incidental food contact. Hence, it is appropriate for biomedical applications.

The supply oil pressure from the compressor is 25bar. A directional valve regulates oil flow and, thus, controls the movement of the actuation cylinder. Two pressure sensors were mounted on the valve manifold. Oil is nearly incompressible and the actuation system is not back-drivable, i.e., the piston cannot be easily moved when the directional valve is closed.

For pneumatic actuation, the supply air pressure is 4 bar. Both flow control and pressure control can be implemented. Pressure control is considered superior to flow control to overcome limitations of compressibility, friction and external disturbances [12]. In our application the manipulandum interacts with human subjects and the interaction force varies within a large range, so that we preferred pressure control. For each cylinder chamber, one valve regulates the pressure with the feedback from a pressure sensor.

The hydraulic and pneumatic transmission hoses between the control valves and the cylinders are $6 \mathrm{~m}$ and $5 \mathrm{~m}$ long, respectively. The valves were located at the corner of the scanner room, far from the scanner isocenter. The scanner magnetic field decreases rather quickly with increasing distance from the scanner bore and comes to be only $0.2 \mathrm{mT}$ at the valve location [20] (For comparison, the magnetic field of the earth is about $0.06 \mathrm{mT}$ ). This special setup, different from conventional fluidic actuators, was taken to fulfill the fMRI-compatibility requirements.

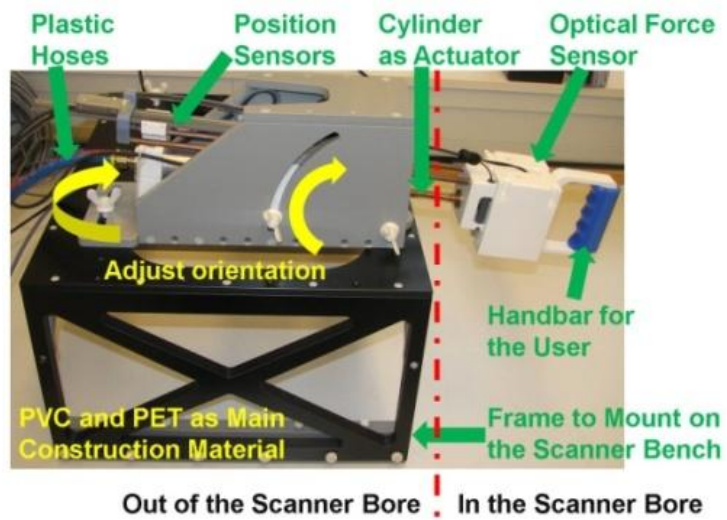

Fig. 3 Composition of the fMRI-compatible haptic interface

\section{D. fMRI-Compatibility Test}

The fMRI-Compatibility of the two robotic systems must be examined by fMRI experiments. The working position of robots has already been shown by Fig. 1 and Fig. 3. The test consists of three parts:

1. No force or torque is induced by the magnetic fields and the robotic system when it is placed at the working position.

2. The robotic system functions properly as designed when it is placed at the working position. All components work properly, and the whole system can perform the desired movements.

3. The robotic system does not deteriorate fMRI image quality. A mineral water phantom is to be scanned in each of the following experimental conditions: 1) No device, in which the robotic system is not placed into the MRI room; 2) silent device, in which the robotic interface is at the working position but not in operation; 3 ) functioning device, in which the robotic system is at the working position and in operation.

Two methods are taken to evaluate whether artifacts have been introduced into the fMRI images [19]. The signal-to-noise ratio (SNR) in $\mathrm{dB}$ [21]

$$
20 \log _{10}\left(\frac{0.66 \times \text { mean signal }}{\text { Average of noise region standard deviations }}\right)
$$

quantitatively estimates whether additional noise has been introduced into fMRI procedures by the robot. We define the SNR variation threshold to be 5\%. A second method is image subtraction, which qualitatively checks whether image shift or deformation has occurred.

\section{CONTROLlER DESIGN}

\section{A. Hydraulic}

Hydraulic oil compressibility is characterized by the bulk modulus $K$. Changes of pressures $P_{1}$ and $P_{2}$ in the cylinder chambers can be written as

$$
\dot{P}_{k}=\frac{K}{V_{k}}\left(-\dot{V}_{k}+q_{k}\right), k=1,2
$$

Here $V_{1}=V_{10}+x A_{1}$ and $V_{2}=V_{20}+(L-x) A_{2}$ are the total fluid volumes on two sides of the cylinder, $L$ is the stroke of the cylinder, $x$ is the position of the piston, $V_{10}$ and $V_{20}$ are the dead volumes, $A_{1}$ and $A_{2}$ are the cross sections of cylinder chambers, $q_{1}$ and $q_{2}$ are oil flows that are dependent on the chamber oil pressure, supply oil pressure or reservoir oil pressure, and also on the control signal $u$ [22].

From equations (1), the piston velocity can be derived as:

$$
\dot{x}=\frac{1}{A_{1}} q_{1}-x \frac{\dot{P}_{1}}{K}-\frac{V_{10}}{A_{1}} \frac{\dot{P}_{1}}{K}-\frac{1}{A_{1}} \dot{V}_{10}
$$

or

$$
\dot{x}=-\frac{1}{A_{2}} q_{2}+(L-x) \frac{\dot{P}_{2}}{K}+\frac{V_{20}}{A_{2}} \frac{\dot{P}_{2}}{K}+\frac{1}{A_{2}} \dot{V}_{20}
$$

First, we consider the steady situation. Pressure changes and dead volume variations are ignored. In this case, $\dot{P}_{1}, \dot{P}_{2}$ and $\dot{V}_{10}, \dot{V}_{20}$ are all equal to zero. Thus, the velocity of the piston is fully determined by the oil flows $q_{1}$ and $q_{2}$ :

$$
\dot{x}=\frac{1}{A_{1}} q_{1}=-\frac{1}{A_{2}} q_{2}
$$

When the piston moves at a constant speed, the pressures $P_{1}$ and $P_{2}$ are both constants, too. Thus, the oil flows $q_{1}$ and $q_{2}$ only depend on the proportional valve. The control voltage to the proportional valve regulates the piston velocity, and this can be modeled as a lookup table. To deal with model uncertainties, external disturbances, and compliance from the hydraulic system, a velocity controller was designed which consists of a compliance compensation term and a proportional term. 
In our hydraulic system, compliance comes from pressure variations $\dot{P}_{1}, \dot{P}_{2}$, long hose volumes $V_{10}, V_{20}$ and their variations $\dot{V}_{10}, \dot{V}_{20}$. It can significantly affect the system performance. The long hoses are the main source of high compliance. Additionally, it can be observed by visual inspection that the hose volumes also change as the inside pressures change, but this change cannot be well detected. We design the compliance compensation component as:

$$
\dot{x}_{c}=-\frac{c}{2}\left[-x \frac{\dot{P}_{1}}{K}-\frac{V_{10}}{A_{1}} \frac{\dot{P}_{1}}{K}+(L-x) \frac{\dot{P}_{2}}{K}+\frac{V_{20}}{A_{2}} \frac{\dot{P}_{2}}{K}\right]
$$

Here, $c \in[0,1]$ determines to which extent the velocity is compensated. The model errors, external disturbances as well as uncompensated compliance components, were handled by the proportional control term. The proportional term was determined by experiments. The user force $F_{h}$ affects pressures $P_{1}$ and $P_{2}$, and causes a shift in the voltage-velocity lookup table, which gets corrected by the velocity controller.

A PD position controller was designed to work in cascade with the velocity controller to guide the user's hand and track the given position trajectory (Fig. 4). It is not possible to realize impedance control on the hydraulic system, because it is not backdrivable due to the incompressibility of oil. However, the virtual spring for user active movements can be simulated by the following admittance control law (Fig. 4):

$$
\dot{x}=\frac{1}{K_{v}}\left[F_{h}-K_{x}\left(x-x_{0}\right)\right]
$$

Since the manipulandum moves in a low speed range, we can set $K_{v}$ to be small such that the viscous term $K_{v} \dot{x}$ is relatively insignificant in the admittance relationship:

$$
F_{h}-K_{x}\left(x-x_{0}\right)=K_{v} \dot{x} \approx 0,
$$

and the hydraulic system behaves like a virtual spring with stiffness $K_{x}$. Here $K_{v}$ was experimentally defined to be 2 $\mathrm{N} /(\mathrm{cm} / \mathrm{s})$, and $K_{x}$ is $3 \ldots 30 \mathrm{~N} / \mathrm{cm}$. If $K_{x}$ was set to be very small to simulate a soft spring, the term $K_{x}\left(x-x_{0}\right)$ goes close to $K_{v} \dot{x}$, and the viscous effect becomes obvious. With these parameters the system remained stable.

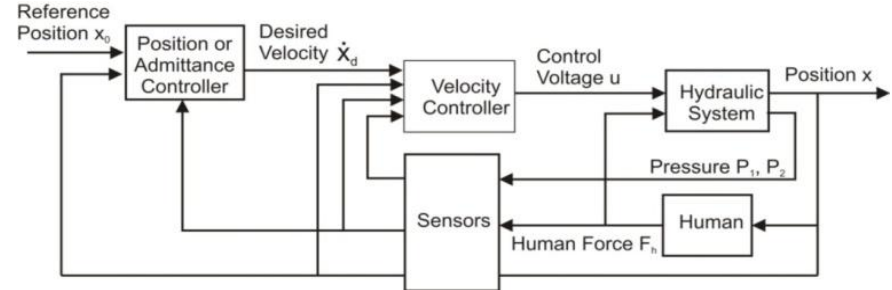

Fig. 4 Hydraulic system Control: position control can be achieved by a PD position controller in cascade with a velocity controller, and the virtual spring can be achieved by setting the virtual admittance as Eq. (4)

Table 2 Parameters for the hydraulic and pneumatic systems

\begin{tabular}{|l|r|c|}
\hline Properties & \multicolumn{1}{|l|}{ Hydraulic } & Pneumatic \\
\hline Power generation & \multicolumn{2}{|l|}{} \\
\hline Supply pressure $P s$ & $15 / 25$ bar* & 4 bar* \\
\hline Exhaust pressure $P e$ & 0.4 bar* & $0^{*}$ \\
\hline
\end{tabular}

\begin{tabular}{|l|c|c|}
\hline Fluid media \\
\hline Bulk modulus $K$ & $1.25 \times 10^{4} \mathrm{bar}$ & Pressure $P$ \\
\hline Density $\rho$ & $856 \mathrm{~kg} / \mathrm{m}^{3}$ & $P / P e \times 1.2 \mathrm{~kg} / \mathrm{m}^{3}$ \\
\hline Kinetic viscosity $v$ & $3.1 \times 10^{-5} \mathrm{~m}^{2} / \mathrm{s}$ & $1.5 \times 10^{-5} \mathrm{~m}^{2} / \mathrm{s}$ \\
\hline Double acting cylinder \\
\hline Cross section $A_{l}$ & $2.54 \mathrm{~cm}^{2}$ & $9.62 \mathrm{~cm}^{2}$ \\
\hline Cross section $A_{2}$ & $1.41 \mathrm{~cm}^{2}$ & $7.85 \mathrm{~cm}^{2}$ \\
\hline Stroke $L$ & $0.24 \mathrm{~m}$ & $0.25 \mathrm{~m}$ \\
\hline Work pressure limit & $25 \mathrm{bar}$ & $6 \mathrm{bar}$ \\
\hline Transmission Hose & $6 \mathrm{~m}$ & $5 \mathrm{~m}$ \\
\hline Length $L_{t}$ & \multicolumn{3}{|l}{} \\
\hline Cross section $A_{t}$ & $0.317 \mathrm{~cm}$ & $0.283 \mathrm{~cm}$ \\
\hline Dynamics & $-194 \ldots 356 \mathrm{~N}$ & $-314 \ldots 384 \mathrm{~N}$ \\
\hline Force range & $-0.2 \ldots 0.3 \mathrm{~m} / \mathrm{s}$ & $-1.51 \ldots 1.67 \mathrm{~m} / \mathrm{s}$ \\
\hline Velocity range
\end{tabular}

*Pressure value relative to environmental pressure 1.013bar

\section{B. Pneumatic}

Since the pressure sensor measures the cylinder pressure relative to the environmental pressure, we also use relative pressure. The force by the pneumatic cylinder is

$$
F_{c}=P_{1} A_{1}-P_{2} A_{2} .
$$

Here, we regulate the pressures $P_{1}$ and $P_{2}$ in two cylinder chambers by two independent valves, and thus regulate the force produced by the cylinder.

Given the desired force $F_{d}$, the desired pressures $P_{l d}$ and $P_{2 d}$ are calculated in the following way:

$$
\left\{\begin{array}{l}
P_{1 d}=\frac{1}{A_{1}}\left(F_{0}+\max \left(F_{d}, 0\right)\right) \\
P_{2 d}=\frac{1}{A_{2}}\left(F_{0}+\max \left(-F_{d}, 0\right)\right)
\end{array}\right.
$$

Here, $F_{0}$ is the minimum chamber force. A first order controller was designed for pressure control:

$$
u_{1,2}=\frac{2}{\frac{1}{2 \pi \times f} s+1} e_{P}
$$

And $e_{P}$ is the pressure error. The pressure control loop is the inner-most loop of the pneumatic system for both position and impedance control. Then, we close the force control loop for force and impedance control, and close the position loop for position control.

A PD position controller with friction compensation worked in cascade with the force-pressure regulator to obtain user passive movement.

$$
F_{d}=k_{v}\left(\dot{x}_{0}-\dot{x}\right)+k_{x}\left(x_{0}-x\right)-F_{h}-F_{f}
$$

Due to manufacture and material properties, the friction force $F_{f}$ depends not only on velocity, but also on position. The friction was modeled as the summation of velocity related and position related friction forces, and then compensated by force-pressure control. The user force was measured by the optical force sensor and got corrected afterwards.

Both admittance control and impedance control can be implemented on the pneumatic system $[23,24]$ to simulate the 
spring. Admittance control requires a good position/velocity controller that is robust against force disturbances, as the velocity controller in our hydraulic system. Here the position controller depends on the nested force-pressure regulator and suffers from the long distance between the valves, pressure sensors and the cylinder. Thus, admittance control is not the optimal option. Besides, pneumatic systems are natural impedances due to the compressibility of air, and impedance control can be realized directly by pressure regulation.

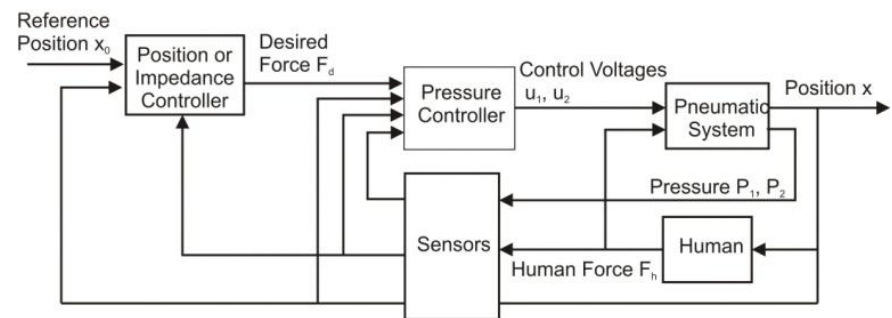

Fig. 5 Pneumatic position control can be achieved by a PD position controller in cascade with a pressure controller, and the virtual spring can be achieved by setting the virtual impedance as Eq. (8)

The impedance control law is quite straightforward

$$
F_{d}=-K_{x}\left(x-x_{0}\right) \text {. }
$$

It calculates the desired force from the measured position and the specified stiffness, and then feed this signal to force-pressure regulation to achieve the desired force.

\section{Control Software and Data Acquisition}

The controllers were implemented in MATLAB Simulink and then compiled to a computer that runs an XPC target and communicates with the system by a data acquisition card (AD614, HUMUSOFT). The sampling frequency was $1 \mathrm{kHz}$.

\section{RESULTS AND DISCUSSION}

\section{A. fMRI-Compatibility Evaluation}

Both robotic systems were tested in a $3.0 \mathrm{~T}$ MRI system (Philips Medical Systems, Eindhoven, The Netherlands), with a phantom imaging object [19]. Their working position is not in the imaging area.

1. No force or torque was observed when the hydraulic or pneumatic system was placed at their working position.

2. The force and positions sensors worked properly. Both robotic systems functioned properly when they were placed at the working position, as will be shown in the next subsections.

3. In each of the three experimental conditions, the phantom body was imaged as 31 slices. 20 images were acquired for every slice of the phantom. Since slide 31 was closest to the robotic systems, the worst image deterioration would happen to this slice. Therefore, we checked slice 31 for SNR and images subtraction.

It has been shown that high SNR values were obtained in all fMRI experiments (Table 3). After introduction of the hydraulic or pneumatic devices into the scanner environment, variations of SNR were all below $5 \%$. The decrease of SNR values were minor and could be attributed to statistical errors.

At slice 31, images from the fMRI experiments on hydraulic as well as pneumatic robotic systems were shown in Fig. 6, together with the subtraction results by the corresponding control image. No significant differences were observed.

The experimental results have verified that both robotic systems are fMRI-compatible.

Table 3 SNR in (dB) for slice 31: Mean (Standard Deviation)

\begin{tabular}{|c|c|c|c|c|}
\hline Condition & \multicolumn{2}{|c|}{ Hydraulic* } & \multicolumn{2}{c|}{ Pneumatic* } \\
\hline No Device & $45.7(0.4)$ & N/A & $47.7(0.4)$ & N/A \\
\hline $\begin{array}{c}\text { Silent } \\
\text { Device }\end{array}$ & $45.6(0.3)$ & $-0.22 \%$ & $47.5(0.5)$ & $-0.42 \%$ \\
\hline $\begin{array}{c}\text { Functioning } \\
\text { Device }\end{array}$ & $45.5(0.5)$ & $-0.44 \%$ & $47.4(0.4)$ & $-0.63 \%$ \\
\hline
\end{tabular}

*: Two experiments were not done at the same day
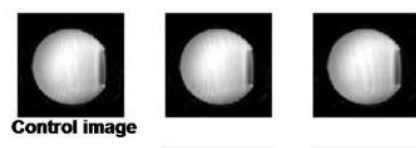

Hydraulic System
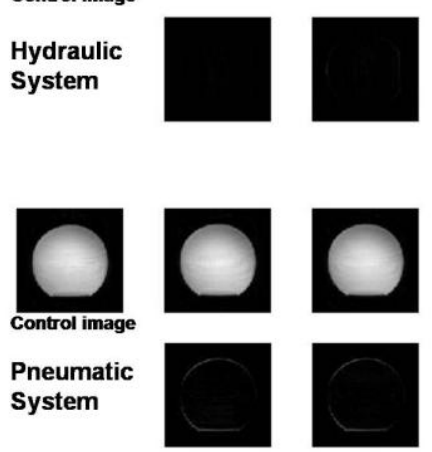

System

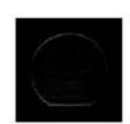

Fig. 6 Phantom images from the fMRI-compatibility test of the hydraulic (upper part) and pneumatic (lower part) robotic systems. For each part, the left image was obtained in the 'No device' condition and taken as the control image. The other two columns of images were obtained for the 'Silent device' and 'Functioning device' conditions, and their difference with the control image by direct subtraction.

\section{B. Control Performance: Hydraulic System}

To analyze the influence of working pressure on the dynamic performance, we tested the hydraulic system at two supply pressures of 15 bar and 25 bar, respectively. Here, 15 bar is the minimal working pressure for the hydraulic system to fulfill the defined velocity requirement, while 25 bar is the limit pressure for the hydraulic system to work safely.

The position control performance was first examined for step responses (Fig. 7). The reference step curve jumped twice from $5 \mathrm{~cm}$ to $15 \mathrm{~cm}$ and back, and then jumped twice from $5 \mathrm{~cm}$ to 10 $\mathrm{cm}$ and back. When the hydraulic system worked at 15 bar, the steady position error was smaller than $0.06 \mathrm{~cm}$, overshoot was smaller than $0.02 \mathrm{~cm}$, and rise time was about $3.14 \mathrm{~s}$. When the hydraulic system worked at 25 bar, the steady position error was still smaller than $0.06 \mathrm{~cm}$, but the overshoot went up to $0.27 \mathrm{~cm}$ and the rise time decreased to $0.86 \mathrm{~s}$. 


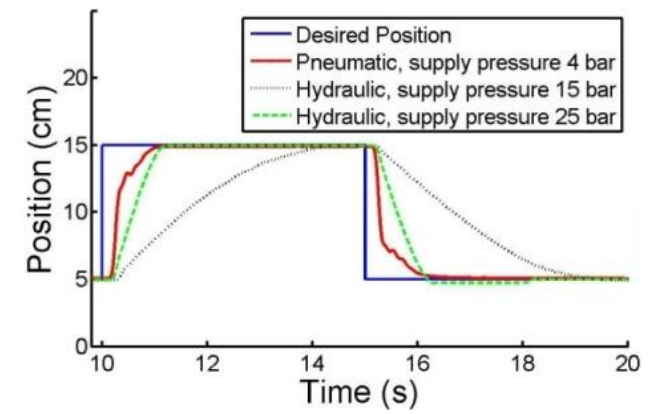

Fig. 7 Position control: step responses of the robotic systems

We then checked the position controlled hydraulic system for dynamic tracking performance. A so-called "chirp" signal from MATLAB Simulink was taken as the reference trajectory (Fig. 8). The signal was of sinusoidal shape, fixed amplitude of $10 \mathrm{~cm}$, and offset $12 \mathrm{~cm}$. The frequency of this signal linearly increased from 0 to $1 \mathrm{~Hz}$ as time went from 0 to $100 \mathrm{~s}$. The actual position curve was recorded and compared with the reference "chirp" signal for bandwidth information (Fig. 8). The position bandwidth for the given signal was $0.48 \mathrm{~Hz}$ when the hydraulic system worked at 15 bar, and went up dramatically to $0.65 \mathrm{~Hz}$ for the working pressure of $25 \mathrm{bar}$.
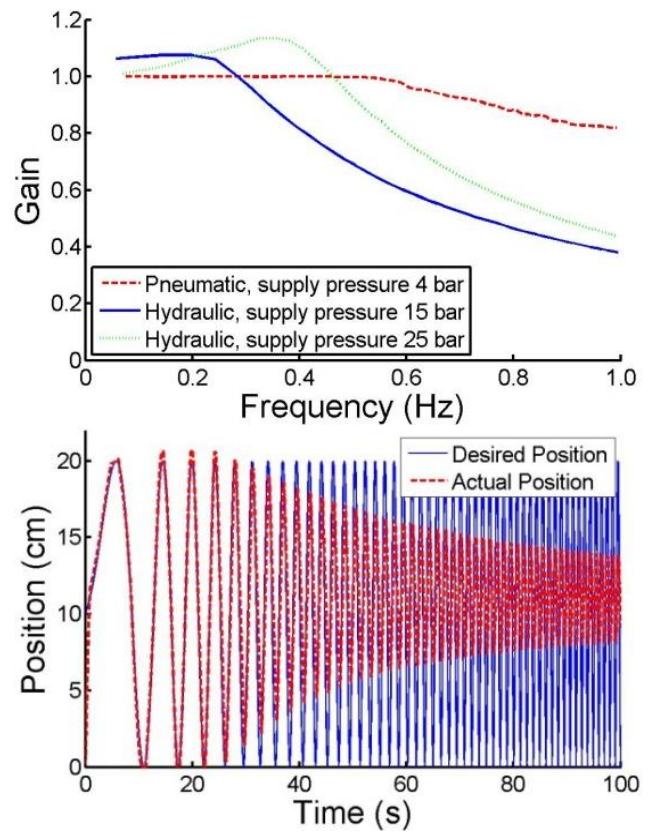

Fig. 8 Position control bandwidth of the robotic systems

User active movements were achieved by the simulated virtual spring. Fig. 9 shows an example spring of stiffness 5 $\mathrm{N} / \mathrm{cm}$ when the hydraulic system worked at 15 bar and 25 bar of supply pressure. In the ideal case, the computed virtual force should equal to the user force. That is, $F_{h}=F_{v}=K_{x}\left(x-x_{0}\right)$. It can be seen from the plot that the virtual force curve coincided quite well with the user force curve at 25 bar working pressure, and was slightly postponed at 15 bar working pressure. When the spring constant is small to simulate a soft spring or the device moves fast, the neglected viscous term becomes significant and blurs the spring feeling. This resulted from the admittance control law we used.

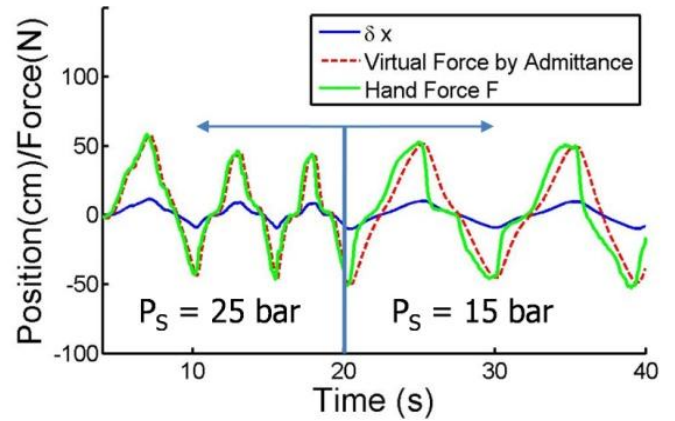

Fig. 9 Virtual spring simulation: an example

\section{Control Performance: Pneumatic System}

We used exactly the same procedures to analyze the controlled performance of the pneumatic system as we did with the hydraulic system. According to the step responses (Fig. 7), the steady position error was smaller than $0.25 \mathrm{~cm}$, overshoot smaller than $0.01 \mathrm{~cm}$, and the rise time was about $0.86 \mathrm{~s}$. The position bandwidth for the given "chirp" signal was around 0.9 $\mathrm{Hz}$, higher than the bandwidth of the hydraulic system working at 15 bar or 25 bar.

The simulated spring was achieved by controlling the cylinder force $F_{c}$ to closely follow the desired virtual force by the impedance control law $-K_{x}\left(x-x_{0}\right)$. The spring effect was more natural and obvious than that of the hydraulic system.

\section{Comparison of the Two fMRI-Compatible Systems}

We summarize the characteristics of hydraulic and pneumatic actuation in Table 4.

Table 4 Comparison of hydraulic and pneumatic actuation for fMRI-compatible applications

\begin{tabular}{|c|c|c|}
\hline Aspects & Hydraulic Actuation & Pneumatic Actuation \\
\hline \multicolumn{3}{|c|}{ fMRI-Compatibility and Related Challenges } \\
\hline Fluid media & \multicolumn{2}{|c|}{ Oil and air are both magnetically inert } \\
\hline Cylinder & \multicolumn{2}{|c|}{$\begin{array}{l}\text { MRI-compatible materials such as Bronze, } \\
\text { aluminum, plastic, etc. }\end{array}$} \\
\hline Hose length & \multicolumn{2}{|c|}{$\begin{array}{l}\text { - } \geq 5 \mathrm{~m} \\
\text { Active components (e.g., valves) are far from } \\
\text { the scanner for fMRI-compatibility } \\
\text { - } \quad \text { This increases compliance of the system } \\
\text { - } \quad \text { Pressure sensors are far away from actuator, } \\
\text { causing inaccuracies and time delay }\end{array}$} \\
\hline \multicolumn{3}{|l|}{ Fluid Power } \\
\hline Power generation & Compressor & Compressor \\
\hline Flow & Laminar & Laminar \& turbulent \\
\hline Working pressure & $\geq 15$ bar* & $\leq 6$ bar* \\
\hline Force & Large & Medium \\
\hline \multicolumn{3}{|l|}{ Working Mode } \\
\hline Component & Directional valve & Pressure regulation valve \\
\hline Control target & $\begin{array}{l}\text { Flow control, regulate } \\
\text { velocity and position }\end{array}$ & $\begin{array}{l}\text { Pressure control, regulate } \\
\text { force }\end{array}$ \\
\hline Position control & $\begin{array}{l}\text { High accuracy } \\
\text { Low bandwidth }\end{array}$ & $\begin{array}{l}\text { Medium accuracy } \\
\text { Medium bandwidth }\end{array}$ \\
\hline Velocity range & Small & Big \\
\hline $\begin{array}{l}\text { Friction or } \\
\text { force disturbances }\end{array}$ & Robust & Sensitive \\
\hline Back drivability & Not backdrivable & backdrivable \\
\hline \multicolumn{3}{|l|}{ Others } \\
\hline Leakage & Rare & Not a problem \\
\hline Complexity\&Cost & High & Medium \\
\hline Maintenance & Medium & Simple \\
\hline
\end{tabular}

*: Relative to environment air pressure. 
The design requirements have been fulfilled by both the hydraulic system and the pneumatic system with different working pressures. With the hydraulic system, we were able to achieve smoother movements, higher position control accuracy and improved robustness against force disturbances than with the pneumatic system. In contrast, the pneumatic system is backdrivable and shows better and faster force control performance. Furthermore, it is easier to maintain and has no serious consequences by leakages. In general, pneumatic actuation is more favorable for fast or force-controlled fMRI-compatible applications, whereas hydraulic actuation can be recommended for applications that require higher position accuracy and slow and smooth movements.

\section{CONCLUSION}

We conclude that both hydraulic and pneumatic actuation systems can be developed for fMRI-compatible applications. The fMRI-compatibility requirements can be fulfilled by special selection of materials and a nonconventional fluidic setup. The resulted limiting factors, such as long distance between cylinders and valves/pressure sensors, long transmission hoses as well as the usage of second quality fMRI-compatible components, increased control difficulties. Nevertheless, satisfactory control performances have been achieved by classical control strategies. Performances of the hydraulic and pneumatic actuation systems differ from each other due to the different physical properties of oil and air. The user has to decide, which system better fits the requirements of a specific application.

In future, stability and robustness of the system during robot-human interaction still need further study.

\section{ACKNOWLEDGMENT}

The authors would like to thank our colleagues Dr. Tobias Nef, Mr. Joachim von Zitzewitz, Mr. Severin Eisner and Mr. Andreas Brunschweiler for their support for this work. The authors would also thank Prof. Dr. Peter Bösiger, Dr. Roger Lüchinger and the MR center of ETH and University Zurich for providing the MRI scanner facility.

This work was supported in part by the Swiss National Science Foundation NCCR on Neural Plasticity and Repair, project P8 Rehabilitation Technology Matrix as well as ETH Research Grant TH-34 06-3 MR-robotics

\section{References}

N. V. Tsekos, A. Khanicheh, E. Christoforou, and C. Mavroidis, "Magnetic Resonance-Compatible Robotic and Mechatronics Systems for Image-Guided Interventions and Rehabilitation: A Review Study," Аппи Rev Biomed Eng, vol. 9, pp. 351-387, 2007.

[2] N. Yu and R. Riener, "Review on MR-compatible robotic systems," in IEEE/RAS-EMBS International Conference on Biomedical Robotics and Biomechatronics Pisa, Italy, 2006.

[3] G. S. Harrington, C. T. Wright, and J. H. Downs, 3rd, "A new vibrotactile stimulator for functional MRI," Hum Brain Mapp, vol. 10, pp. 140-5, Jul 2000.

[4] S. M. Golaszewskir, C. M. Siedentopf, E. Baldauf, F. Koppelstaetter, W. Eisner, J. Unterrainer, G. M. Guendisch, F. M. Mottaghy, and S. R. Felber, "Functional Magnetic Resonance Imaging of the Human Sensorimotor Cortex Using a Novel
Vibrotactile Stimulator," in Neuroscience Letters. vol. 17, 2002, pp. 421-430.

[5] R. Gassert, L. Dovat, G. Ganesh, E. Burdet, H. Imamizu, T. Milner, and H. Bleuler, "Multi-joint arm movements to investigate motor control with fMRI," Conf Proc IEEE Eng Med Biol Soc, vol. 5, pp. 4488-91, 2005.

[6] A. Khanicheh, A. Muto, C. Triantafyllou, B. Weinberg, L. Astrakas, A. Tzika, and C. Mavroidis, "fMRI-compatible rehabilitation hand device," J Neuroengineering Rehabil, vol. 3, p. 24, 2006.

[7] R. Riener, T. Nef, and G. Colombo, "Robot-aided neurorehabilitation of the upper extremities," Med Biol Eng Comput, vol. 43, pp. 2-10, Jan 2005.

[8] M. Flueckiger, M. Bullo, D. Chapuis, R. A. G. R. Gassert, and Y. A. P. Y. Perriard, "fMRI compatible haptic interface actuated with traveling wave ultrasonic motor," in Industry Applications Conference, 2005. Fourtieth IAS Annual Meeting. Conference Record of the 2005, 2005, pp. 2075-2082 Vol. 3.

[9] R. Gassert, R. Moser, E. Burdet, and H. Bleuler, "MRI/fMRI-compatible robotic system with force feedback for interaction with human motion," IEEE-ASME Transactions on Mechatronics, vol. 11, pp. 216-224, Apr 2006.

[10] R. Riener, T. Villgrattner, R. Kleiser, R. Nef, and S. Kollias, "fMRI-Compatible Electromagnetic Haptic Interface," in Proc. IEEE/EMBS Annual International Conference, 2005.

[11] D. Chapuis, R. Gassert, L. Sache, E. Burdet, and H. Bleuler, "Design of a Simple MRI/fMRI Compatible Force/Torque Sensor," in Proc. IEEE International Conference on Robotics and Intelligent Systems, 2004.

[12] J. M. Hollerbach, I. W. Hunter, and J. Ballantyne, "A comparative analysis of actuator technologies for robotics," in The robotics review 2: MIT Press, 1992, pp. 299-342.

[13] R. Gassert, A. Yamamoto, D. Chapuis, L. Dovat, H. Bleuler, and E. Burdet, "Actuation methods for applications in MR environments," Concepts in Magnetic Resonance Part B-Magnetic Resonance Engineering, vol. 29B, pp. 191-209, Oct 2006.

[14] J. Diedrichsen, H. Yasmin, R. Tushar, and S. Reza, "Neural Correlates of Reach Errors," in Journal of Neuroscience. vol. 25, 2005, pp. $9919-9931$.

[15] R. W. Briggs, I. Dy-Liacco, M. P. Malcolm, H. Lee, K. K. Peck, K. S. Gopinath, N. C. Himes, D. A. Soltysik, P. Browne, and R. Tran-Son-Tay, "A pneumatic vibrotactile stimulation device for fMRI," in Magnetic Resonance in Medicine. vol. 51, 2004, pp. 640-643.

[16] A. C. Zappe, T. Maucher, K. Meier, and C. Scheiber, "Evaluation of a pneumatically driven tactile stimulator device for vision substitution during fMRI studies," in Magnetic Resonance in Medicine. vol. 51, 2004, pp. 828-834.

[17] D. Kim, E. Kobayashi, T. Dohi, and I. Sakuma, "A new, compact MR-compatible surgical manipulator for minimally invasive liver surgery," Medical Image Computing and Computer-Assisted Intervention-Miccai 2002, Pt 1, vol. 2488, pp. 99-106, 2002.

[18] R. Wang, T. Foniok, J. I. Wamsteeker, M. Qiao, B. Tomanek, R. A. Vivanco, and U. I. Tuor, "Transient blood pressure changes affect the functional magnetic resonance imaging detection of cerebral activation," Neuroimage, vol. 31, pp. 1-11, May 152006.

N. Yu, W. Murr, A. Blickenstorfer, S. Kollias, and R. Riener, "An fMRI compatible haptic interface with pneumatic actuation " in International Conference on Rehabilitation Robotics Nordwijk, The Netherlands, 2007.

Philips, "Technical Description: Intera 1.5T Release 2.5 series, Achieva 1.5T / 3.0T / XR Release 2.5, Panorama HFO Release 2.5 series," Magnetic Resonance 452213268821 , May 2007.

[21] E. Burdet, R. Gassert, G. Gowrishankar, D. Chapuis, and H. Bleuler, "fMRI Compatible Haptic Interfaces to Investigate Human Motor Control," in Experimental Robotics IX, 2006, pp. 25-34.

[22] G. A. Sohl and J. E. Bobrow, "Experiments and simulations on the nonlinear control of a hydraulic servosystem," Control Systems Technology, IEEE Transactions on, vol. 7, pp. 238-247, 1999.

[23] R. Richardson, M. Brown, B. Bhakta, and M. C. Levesley, "Design and control of a three degree of freedom pneumatic physiotherapy robot," Robotica, vol. 21, pp. 589-604, 2003.

[24] Z. Yong and E. J. Barth, "Impedance Control of a Pneumatic Actuator for Contact Tasks," in Robotics and Automation, 2005. ICRA 2005. Proceedings of the 2005 IEEE International Conference on, 2005, pp. 987-992. 